\title{
Initiation of microcalli in culture of pea (Pisum sativum L.) isolated microspores
}

\author{
Sergey V. Bobkov a, \\ a All-Russia Research Institute of Legumes and Groat Crops, Orel Region, 302502, Russia
}

Accepted 5th January 2017

\begin{abstract}
Innovative biotechnologies based on use of double haploids enables developing new varieties considerably faster compared to conventional plant breeding approaches. In pea, reliable methods of haploid plants production are not fully elaborated yet. The current research aimed at testing different conditions (genotype, medium, and stress treatment) for initiation of sporophytic developmental shift in culture of pea isolated microspores. Reprogramming pea microspores towards a sporophytic development was stimulated with temperature stress. Cold $\left(+4^{\circ} \mathrm{C}\right)$ and heat $\left(+35^{\circ} \mathrm{C}\right)$ stress treatments were applied to pea isolated flower buds and microspores, respectively. Microspores were isolated from plants of 6 pea genotypes, treated at 18 temperature regimes and cultivated in 8 liquid nutrient media with various chemical compounds including growth regulators, vitamins, sugars, glutamine, casein hydrolysate, and osmotic agents. Microcalli were produced from isolated microspores of pea breeding line 109b and variety Stabil in conditions of nutrient media KM-ap1 and MSB-M3 after cold $\left(+4^{\circ} \mathrm{C}\right)$ stress treatment for 16 and 10 days, respectively. The media KM-ap1 and MSB-M3 contained a relatively low concentration of sugar (10 and $6 \mathrm{~g} \mathrm{~L}^{-1}$, respectively), and were supplemented with polyethylene glycol 6000 or mannitol as osmotic agents.
\end{abstract}

Keywords: medium, microcallus, microspore, pea, sporophyte, stress

\section{INTRODUCTION}

In most species, including legumes, breeders use self-pollination or backcrossing over many generations for obtaining true-breeding lines with high level of homozygosity. Alternatively, completely (at all loci) homozygous lines with various sets of alleles could be obtained from reprogrammed microspores for a single generation (Dwivedi et al., 2015). Application of haploidy in pea breeding could significantly reduce time and material consumption for development of new varieties as dihaploid plants may be implicated on various stages of pea breeding. Use of haploidy in pea introgressive hybridization could facilitate transfer of agronomic value alleles into elite genotypes (Bobkov and Lazareva, 2012) and haploid plants may also facilitate genetics researches.

Commonly, haploid plants are obtained via androgenesis, gynogenesis, parthenogenesis, and genome elimination. Androgenesis is based on use of two approaches, in vitro cultivation of anthers or isolated microspores. These approaches are usually used in the Fabaceae (Lulsdorf et al., 2011).

Cultivation of pea isolated anthers stressed by low or high temperatures led to appearance of morphogenic and embryogenic calli, and also regenerated plants (Sidhu and Davies, 2005;

\footnotetext{
* Author for correspondence: Sergey V. Bobkov, 302502, Russia, Orel Region, Pos. Streletsky, Molodezhnaya Street, H. 10, Build. 1. Email svbobkov@gmail.com
} 
Bobkov, 2010, 2014). Anthers used in experiments consisted of microspores and somatic tissues that complicate the interpretation of results. For example, in culture of pea isolated anthers active proliferation of somatic tissues and subsequent regeneration of plants from somatic cells but not from microspores was revealed (Bobkov, 2010).

Isolated microspores are considered as more attractive tool for investigation of pea haploidy. In culture of isolated microspores symmetrical division of nuclei, a marker of sporophytic developmental shift was initiated after applying cold and heat stress treatments (Croser and Lulsdorf, 2004; Croser et al., 2005). In vitro cultivated microspores with symmetrical divisions of nuclei and subsequent embryogenesis were also observed (Croser et al., 2006). Pyramiding of multiple (cold, electroporation, osmotic) stress treatments promoted microspore reprogramming (Ochatt et al., 2009). Flow cytometry clearly detected embryogenic divisions within isolated microspores after their pretreatments with combined stresses (Ribalta et al., 2012). A reduced number of haploid plants were recovered from isolated microspores through organogenesis from microspore-derived calli and via embryogenesis from microspores (Ochatt et al., 2009; Lulsdorf et al., 2011).

Highly efficient methods to obtain pea haploid plants as needed for their subsequent exploitation within a breeding context are not yet available. Knowledge about the sporophytic developmental reprogramming of legume microspore is still incomplete (Ochatt et al., 2009) although additional information has continued appearing (Ribalta et al., 2012). Accordingly, the objective of the current research was that of contribution to this search of understanding conditions (genotype, stress treatment, medium) which could lead to sporophytic developmental shift in culture of pea isolated microspores.

\section{MATERIALS AND METHODS}

Microspores of pea (Pisum sativum L.) varieties Faraon (af), Gothic (af), Stabil (af), Vizir (Af, def), Spartak (af, tac) and of the breeding line 109b (af) were used in all experiments (Table 2). Plants of these varieties represented genotypes afila (af), afila tendrilled acacia (af, tac), and seed developed funiculus $(d e f)$.

Viability and developmental stages of microspores was determined after staining in 4\% propionic carmine. Specimens were analyzed under a microscope Axioskop 40 (Karl Zeiss, Germany) and microspores were preferably isolated at various uninucleate stages of development after release from tetrads (Figure 1A and $1 \mathrm{~B})$.

Pea flower buds were surface sterilized with $0.5 \%$ solution of sodium chlorhexidin gluconate for 10 minutes. They were rinsed thrice with sterile deionized water and dried on sterile filter paper inside Petri dishes. Microspores were released after bud homogenization with pestle in porcelain mortar in $2-3 \mathrm{ml}$ of medium $\mathrm{B}$, which contained $1490 \mathrm{mg} \mathrm{L}^{-1} \mathrm{KCl}, 250 \mathrm{mg} \mathrm{L}^{-1} \mathrm{MgSO}_{4} \times$ $7 \mathrm{H}_{2} \mathrm{O}, 140 \mathrm{mg} \mathrm{L}^{-1} \mathrm{KH}_{2} \mathrm{PO}_{4}, 110 \mathrm{mg} \mathrm{L}{ }^{-1} \mathrm{CaCl}_{2} \times$ $2 \mathrm{H}_{2} \mathrm{O}$, and $54.7 \mathrm{~g} \mathrm{~L}^{-1}$ D-mannitol ( $\mathrm{KyO}$ and Harada, 1986). The resulting homogenate was successively sieved through both a $100 \mu \mathrm{m}$ and a $40 \mu \mathrm{m}$ nylon filter (Millipore, USA). The microspore suspension was rinsed with medium $\mathrm{B}$ on a $15 \mu \mathrm{m}$ nylon filter for the further removal of small debris particles (Croser et al., 2006). Isolated microspores were washed thrice by centrifugation in $1.5 \mathrm{ml}$ tubes for 15 minutes at $100 \mathrm{~g}$. The supernatant was discarded and the pellet re-suspended in $1 \mathrm{ml}$ of rich liquid nutrient media at a density of $10^{5}$ microspores per $\mathrm{ml}$ (Ochatt et al., 2009). All the above mentioned procedures were carried out under sterile conditions using a laminar air flow cabinet. Isolated microspores were cultivated in $10 \times 35$ $\mathrm{mm}$ glasses at $25^{\circ} \mathrm{C}$ and a 16 hour light photoperiod (flux density of about $250 \mu \mathrm{mol} \mathrm{m}{ }^{-2}$ $\left.\mathrm{s}^{-1}\right)$ in 8 liquid nutrient media (Table 1).

Experimental nutrient media were KM (Kao and Michayluk, 1980), NLN (Lichter, 1982), or MSB media, which is modified MS (Griga et al., 1986), as detailed in Table 1. They contained 100 $\mathrm{mg} \mathrm{\textrm {L } ^ { - 1 }}$ myo-inositol, $2 \mathrm{mg} \mathrm{\textrm {L } ^ { - 1 }}$ glycine, were adjusted to $\mathrm{pH} 5.8$ and differed in terms of the presence and quantity of growth regulators such as 2,4-D (2,4-dichlorophenoxyacetic acid), BA (6benzyladenine), NAA ( $\alpha$-naphthaleneacetic acid), IBA (indole-3-butyric acid), vitamins (thiamine, nicotinic acid, pyridoxine), sugars (sucrose, 
mannitol), polyethylene glycol 6000 (PEG), glutamine, and casein hydrolysate.

Nutrient media MSB contained MS salts (Murashige and Skoog, 1962) and vitamins of medium B5 (Gamborg and Eveleigh, 1968). As compared to the original $\mathrm{Kao}$ and Michayluk (1980) formulation, all KM-based media did not contain amino acids except glycine, and were supplemented with vitamins of B5 (Gamborg et al., 1968) medium instead of their original composition. Variants of medium KM contained PEG as osmotic agent. Media NLN-M1 and NLN-M2 only differed in the concentration of sucrose (Table 1), so that during the first week isolated microspores were cultured in medium NLN-M1 with high sucrose concentration $(170 \mathrm{~g}$ $\mathrm{L}^{-1}$ ) and thereafter on medium NLN-M2 with decreased sucrose content $\left(100 \mathrm{~g} \quad \mathrm{~L}^{-1}\right)$. Reprogramming of pea microspores from gametophytic to sporophytic pathway of development was triggered with use of two temperature stress pretreatments, cold $\left(+4^{\circ} \mathrm{C}\right)$ and heat $\left(+35^{\circ} \mathrm{C}\right)$ of various duration (Table 2$)$.

Cold stress treatment was applied to isolated flower buds. Heat stress was used for treatment of isolated microspores in medium B (Kyo and Harada, 1986). Thus, 8 nutrient media, 18 temperature regimes of stress treatments and 6 genotypes of pea were tested altogether (Table 2). All experiments included three and more replicate glasses with cultured microspores. Appearance of microcalli at least in one replicate was evaluated as a positive result.

Table 1. Composition of nutrient media used for cultivation of pea isolated microspores.

\begin{tabular}{lccccccccc}
\hline \multirow{2}{*}{ Medium } & \multicolumn{4}{c}{$\mathbf{g ~ L}^{-1}$} & \multicolumn{4}{c}{ mg L $^{-1}$} \\
\cline { 2 - 10 } & CH & Sucrose & PEG & Mannitol & Glutamine & IBA & 2,4-D & NAA & BA \\
\hline KM-A5 & 1 & 10 & 140 & 0 & 0.5 & 0 & 0.05 & 0 & 0 \\
KM-A8 & 0.3 & 3 & 130 & 0 & 0.5 & 0 & 0 & 0 & 0 \\
KM-A81 & 0.7 & 17 & 130 & 0 & 0.5 & 1 & 0 & 0 & 0 \\
KM-A9 & 0.3 & 3 & 130 & 0 & 0.5 & 0 & 0.05 & 0 & 0 \\
KM-ap1 & 0.5 & 10 & 130 & 0 & 0.5 & 0 & 0 & 0.25 & 0.2 \\
NLN-M1 & 0.5 & 170 & 0 & 0 & 0 & 0 & 0 & 0.25 & 0.2 \\
NLN-M2 & 0.5 & 100 & 0 & 0 & 0 & 0 & 0 & 0.25 & 0.2 \\
MSB-M3 & 1 & 6 & 0 & 54.7 & 0.5 & 0 & 0.5 & 0 & 0.5 \\
\hline
\end{tabular}

All media contain $100 \mathrm{mg} \mathrm{L}^{-1}$ myo-inositol, $2 \mathrm{mg} \mathrm{L}^{-1}$ glycine, and are adjusted to $\mathrm{pH}=5.8$.
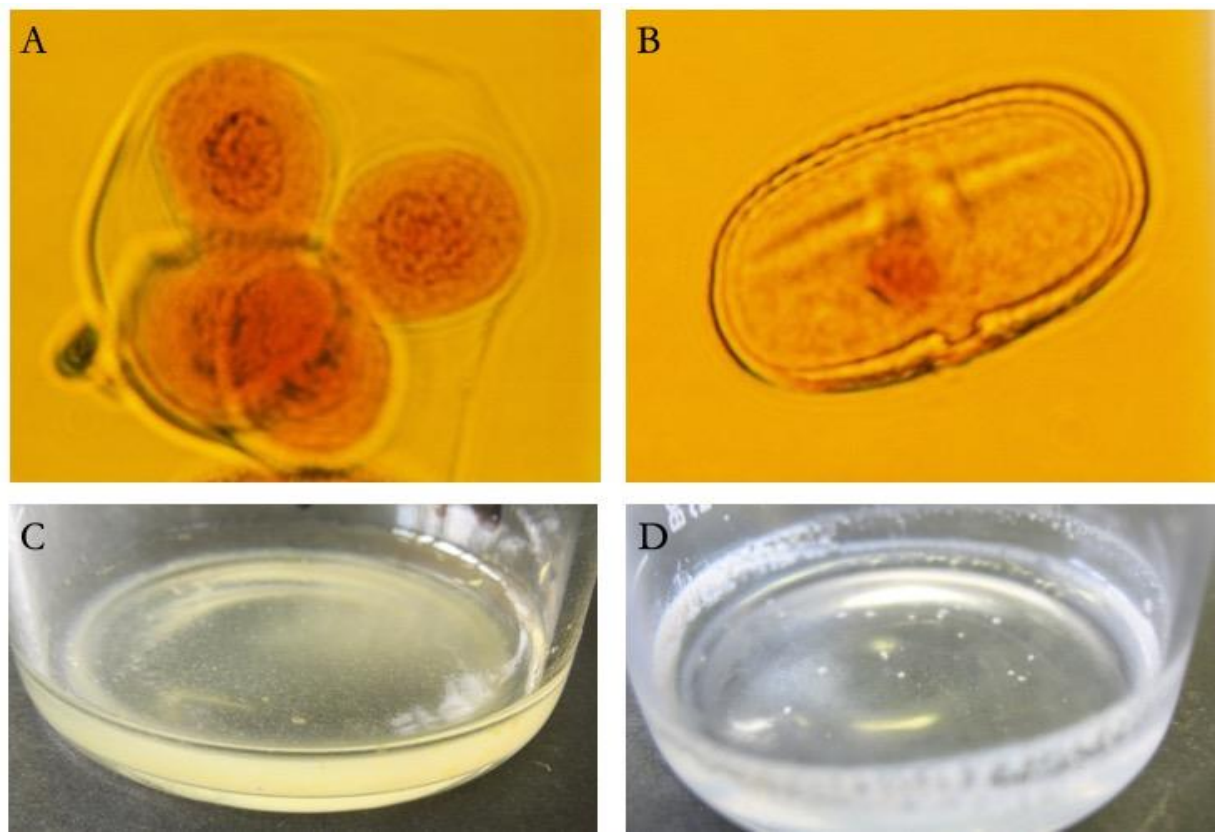

Figure 1. In vitro culture of pea isolated microspores. (A) tetrad of microspores, (B) microspore at miduninucleate stage of development, (C) microcallogenesis in culture of breeding line 109b isolated microspores in conditions of nutrient media KM-ap1, (D) microcallogenesis in culture of variety Stabil isolated microspores in conditions of nutrient media MSB-M3. 
Table 2. Application of various nutrient media, genotypes and temperature stresses for sporophytic reprogramming of pea isolated microspores.

\begin{tabular}{|c|c|c|c|}
\hline \multirow{2}{*}{ Medium } & \multirow{2}{*}{ Variety, breeding line } & \multicolumn{2}{|c|}{ Stress treatment } \\
\hline & & cold $\left(+4^{\circ} \mathrm{C}\right)$, days & heat $\left(+35^{\circ} \mathrm{C}\right)$, hours \\
\hline \multirow{3}{*}{ KM-ap1 } & Spartak (af, tac) & 16 & 0 \\
\hline & \multirow{2}{*}{$109 \mathrm{~b}(a f)$} & 16 & 0 \\
\hline & & 28 & 0 \\
\hline \multirow{7}{*}{ KM-A5 } & Stabil $(a f)$ & 13 & 0 \\
\hline & Gothic $(a f)$ & 14 & 0 \\
\hline & Stabil $(a f)$ & 15 & 0 \\
\hline & $\operatorname{Vizir}(A f, \operatorname{def})$ & 18 & 0 \\
\hline & Gothic $(a f)$ & 19 & 0 \\
\hline & Faraon $(a f)$ & 20 & 0 \\
\hline & Stabil (af) & 21 & 0 \\
\hline \multirow{3}{*}{ KM-A8 } & Gothic $(a f)$ & 4 & 0 \\
\hline & \multirow{2}{*}{ Stabil (af) } & 0 & 18 \\
\hline & & 1 & 18 \\
\hline KM-A81 & Gothic $(a f)$ & 9 & 0 \\
\hline \multirow{3}{*}{ KM-A9 } & \multirow{3}{*}{ 109b (af) } & 4 & 0 \\
\hline & & 2 & 18 \\
\hline & & 6 & 18 \\
\hline \multirow{4}{*}{ NLN-M1 and NLN-M2 } & \multirow{2}{*}{ Gothic $(a f)$} & 7 & 0 \\
\hline & & 12 & 0 \\
\hline & Stabil $(a f)$ & 12 & 0 \\
\hline & Faraon $(a f)$ & 18 & 0 \\
\hline MSB-M3 & Stabil $(a f)$ & 10 & 0 \\
\hline
\end{tabular}

\section{RESULTS AND DISCUSSION}

Microspores of pea varieties Faraon, Gothic, Stabil, Vizir, Spartak, and breeding line 109b were cultured in vitro (Table 2). Cold stress was applied to isolated buds of all genotypes studied. Heat stress as a single treatment was applied only to isolated microspores of variety Stabil. Combined stress treatments (cold plus heat) were consequently directed on isolated buds and microspores for Stabil and breeding line 109b.

Isolated microspores of all pea genotypes were cultured in five variants of KM medium while microspores of varieties Faraon, Gothic, Stabil were tested on media NLM-M1 and NLMM2, and medium MSB-M3 was used for microspore cultures of variety Stabil.

Isolated microspores of pea varieties Stabil, Gothic, Faraon, and breeding line 109b, cultivated in liquid media KM-A5, KM-ap1 and MSB-M3, changed their appearance and exhibited the traits of sporophytic developmental transition as described by Touraev et al. (1996). Some microspores acquired round shape, central position of nucleus, and more intensive coloration of cytoplasm. Protrusion of microplast through apertures of exine was observed among rare number of microspores (Bobkov, 2013).

Microcalli were obtained in isolated microspore culture of breeding line 109b and variety Stabil (Figure 1C and 1D) in two nutrient media, KM-ap1 and MSB-M3 (Table 1). Before microspore isolation, flower buds of pea breeding line $109 \mathrm{~b}$ and variety Stabil had been treated with cold $\left(+4^{\circ} \mathrm{C}\right)$ for 16 and 10 days, respectively.

Effective nutrient media KM-ap1 and MSBM3 contained glutamine, casein hydrolysate and a low concentration of sucrose, but they differed in salt content, osmotic agents and growth regulators, as detailed in Table 1 . On medium KM-ap1, appearance of multiple microcalli 0.5-1 $\mathrm{mm}$ (Figure $1 \mathrm{C}$ ) was observed at $34^{\text {th }}$ day of microspore cultivation after dilution of their suspension with fresh medium at a 1:1 ratio. In nutrient medium MSB-M3 a white suspension appeared after 17 days of microspore cultivation. Dilution of such microspore cultures with fresh 
MSB-M3 medium at a 1:1 ratio resulted 6 days later in formation of rare white microcalli (Figure 1D).

Microcallus production in culture of pea isolated microspores as a preliminary result was reported earlier by Bobkov (2013), but the physiological conditions promoting microspore shift towards a sporophytic pathway of development were not elucidated then. In the current work, various factors influencing microspore reprogramming such as genotype, complete composition of nutrient media, and regimes of stress treatment are assessed (Table 1, 2).

The culture medium formulations used here were selected because they had been used in isolated microspore or protoplast cultures of various species in the past. Thus, nutrient medium MSB (modified MS) is commonly used for in vitro cultivation of various pea tissues including isolated anthers (Griga et al., 1986; Bobkov, 2014). NLN medium showed high efficiency for haploid plant production from isolated microspores of Brassica napus L. (Lichter, 1982), and a modified NLN medium was also successfully used in culture of pea isolated microspores (Ochatt $e t$ al., 2009). On the other hand, KM medium (Kao and Michayluk, 1980) was elaborated especially for cultivation of isolated protoplasts of alfalfa (Medicago sativa L.).

Sucrose and mannitol as carbon source in nutrient media were tested before in the culture of isolated microspores of pea (Ochatt et al., 2009; Lulsdorf et al., 2011). Sucrose at a high concentration yielded better responses than mannitol in terms of microspore-derived calli production. Furthermore, such initiated calli were capable of cell differentiation. However, the stimulating effect of a drop in sucrose concentration from $17 \%$ to $10 \%$ on microspore divisions after a week of cultivation was also reported (Ochatt et al., 2009). Interestingly, the microcalli in this study were initiated in media with a low level of sucrose from the start. Sucrose serves as an energy source and as an osmoticum. Illic-Grubor et al. (1998) used very low concentrations $(0.08-0.1 \%)$ of sucrose and added PEG 4000 as an alternative osmotic agent in microspore cultures of Brassica napus L. On PEG containing medium microspore-derived embryos had two well-developed cotyledons, a proportionate axis and closely resembled their zygotic counterparts developed in ovulo. As discussed by Touraev et al. (1996), sugar starvation at high temperature is a critical factor for reprogramming of wheat microspores to sporophytic pathway of development. Conversely, cultivation of wheat isolated microspores in sucrose rich media resulted in toxic accumulation of starch granules. Possibly therefore, presence of sucrose (as the unique sugar source) in nutrient media is not necessary for the emergence of initial calli. For example, high efficient initial callogenesis was observed in stressed pea anthers on nutrient medium without sucrose but substituted with maltose (Bobkov, 2014).

In this experiment the degree of pea microspore reprograming was not complete. Cells of microcalli seemed to have a limited totipotency. Major part of microcalli did not demonstrate further development towards embryo formation, tissue proliferation and regeneration of plants. Similarly, in cultured non-stressed wheat microspores frequency of multicellular structures was low and majority of them developed into calli which did not regenerate when transferred to a regeneration medium (Indrianto et al., 2001). Optimal stress pretreatment of microspores enhanced number of multicellular structures and led to formation of compact embryos. Therefore, it makes sense to search new stresses and regimes of their application to pea microspore culture for obtaining totipotent tissues and regeneration of haploid plants.

There are many physical factors affecting the acquisition of competence for in vitro regeneration from cultured cells (Ochatt, 2015). But a reduced number of plants, confirmed as haploids by flow cytometry, were obtained from microspores after pyramiding of multiple (cold, electroporation, osmotic) stress treatments (Ochatt et al., 2009). It means that pea microspore needed more prolonged and specific stress treatments to acquire totipotency. In recent research, rare green embryogenic calli and embryos were obtained after stressed at $33^{\circ} \mathrm{C}$ for 2 or 3 days microspores of cultured anthers after their releasing into liquid media with low sucrose content and supplemented with Ficoll or PEG 6000 (unpublished data). Testing new regimes of stress treatments for microspore reprogramming and 
nutrient media for maintenance of embryogenesis could help to elaborate a reliable method of plant regeneration from cultivated pea microspores.

\section{CONCLUSION}

Pea (Pisum sativum L.) is a recalcitrant species for haploid plant production. Reliable methods for obtaining pea haploids are not yet fully elaborated and more research input in this area is needed. In vitro culture of isolated microspore experiments in this work included 6 genotypes of pea, 8 variants of nutrient media, and 18 regimes of temperature stress treatments. Some cultivated microspores exhibited marks of transition to sporophytic pathway of development. Microcalli were initiated in culture of isolated microspores after cold stress treatment in nutrient media KM-ap1 and MSBM3 containing PEG 6000 or mannitol as osmotic agents and a low (10 and $\left.6 \mathrm{~g} \mathrm{~L}^{-1}\right)$ content of sucrose. Major part of microcalli did not demonstrate further development towards differentiation and plant regeneration. Initiation of microcalli is an important preliminary result in terms of media choice for testing in future research. In the next round of experiments combination of factors that led to obtaining microcalli should be repeated and compared with some other new variants. Use of reduced number of pea genotypes could facilitate researches. Replacement of sucrose for maltose would serve as an additional stress (sugar starvation) for improving plant regeneration. In general, appropriate stress treatments together with optimal medium composition would improve pea microspore reprogramming and plant regeneration.

\section{ACKNOWLEDGEMENT}

The author thanks Dr. Galina Suvorova for assistance in conducting of experiments.

\section{REFERENCES}

Bobkov, S. V. 2010. Isolated pea anther culture. Russian Agricultural Sciences 6: 413-416.

Bobkov, S. V. 2013. Reprogrammirovanie izolirovannyh mikrospor goroha na jembriogennyj put' razvitija. Zernoboborye i Krupjanye Kul'tury 3 (7): 5-10 (Bobkov, S. V. 2013. Reprogramming of isolated microspores of pea onto embryogenic pathway of development. Leguminous and Groat Crops 3 (7): 5-10) (in Russian).

Bobkov, S. 2014. Obtaining calli and regenerated plants in anther cultures of pea. Czech Journal of Genetics and Plant Breeding 50 (2): 123-129.

Bobkov, S. V., Lazareva, T. N. 2012. Band composition of electrophoretic spectra of storage proteins in interspecific pea hybrids. Russian Journal of Genetics 48 (1): 47-52.

Croser, J., Lulsdorf, M., Davies, P., Clarke, H., Wilson, J., Sidhu, P., Grewal, R., Allen, K., Damen, T., Warketin, T., Vandenberg, A., Siddique, K. 2005. Haploid embryogenesis from chickpea and field pea - progress towards a routine protocol. In Proceedings of the Australian Branch of the IAPT\& / Perth, Western Australia, 21-24th September, pP 71-82.

Croser, J. S., Lulsdorf, M. M. 2004. Progress towards haploid division in chickpea (Cicer arietinum L.), field pea (Pisum sativum L.) and lentil (Lens culinaris Medik.) using isolated microspore culture. In European Grain Legume Conference, AEP, Dijon, France, p 189.

Croser, J. S., Lulsdorf, M. M., Davies, P. A., Clarke, H. J., Bayliss, K. L., Mallikarjuna, N., Siddique, K. H. M. 2006. Toward doubled haploid production in the Fabaceae: progress, constraints, and opportunities. Critical Reviews in Plant Sciences 25: 139-157.

Dwivedi, S. L., Britt, A. B., Tripathi, L., Sharma, S., Upadhyaya, H. D., Ortiz, R. 2015. Haploids: constraints and opportunities in plant breeding. Biotechnology Advances 33: 812-829.

Gamborg, O., Eveleigh, D. E. 1968. Culture methods and detection of gluconases in cultures of wheat and barley. Canadian Journal of Biochemistry 46 (5): 417-421.

Griga, M., Tejklova, E., Novak, F. J., Kubalakova, M. 1986. In vitro clonal propagation of Pisum sativum L. Plant Cell Tissue and Organ Culture 6: 95-104.

Ilic-Grubor, K., Attree, S. M., Fowke, L. C. 1998. Comparative morphological study of zygotic and microspore-derived embryos of Brassica napus L. as revealed by scanning electron microscopy. Annals of Botany 82 (2): 157-165.

Indrianto, A., Barinova, I., Touraev, A., Heberle-Bors, E. 2001. Tracking individual wheat microspores in vitro: identification of embryogenic microspores and body axis formation in the embryo. Planta 212: 163-174.

Kao, K. N., Michayluk, M. R. 1980. Plant regeneration from mesophyll protoplasts of alfalfa. Z. Pflanzenphysiol. 9: 135141.

Kyo, M., Harada, H. 1986. Control of the developmental pathway of tobacco pollen in vitro. Planta 168: 427-432.

Lichter, R. 1982. Induction of haploid plants from isolated pollen of Brassica napus. Z. Pflanzenthysiol. 105: 427-434.

Lulsdorf, M. M., Croser, J. S., Ochatt S. 2011. Androgenesis and doubled-haploid production in food legumes. Chapter 11. In Biology and Breeding of Food Legumes, ed. A. Pratap, J. Kumar, pp 336-347. Oxfordshire, CABI.

Murashige, T., Skoog, F. 1962. A revised medium for rapid growth and bioassays with tobacco tissue cultures. Physiologia Plantarum 15 (13): 473-497.

Ochatt, S., Pech, C., Grewal, R., Coreux, C., Lulsdorf, M., Jacas, L. 2009. Abiotic stress enhances androgenesis from isolated 
microspores of some legume species (Fabaceae). Journal of Plant Physiology 166: 1314-1328.

Ochatt, S. J. 2015. Agroecological impact of an in vitro biotechnology approach of embryo development and seed filling in legumes. Agronomy for Sustainable Development 35: 535-552.

Ribalta, F., Croser, J., Ochat, S. 2012. Flow cytometry enables identification of sporophytic eliciting stress treatments in gametic cells. Journal of Plant Physiology 169: 104-110.

Sidhu, R., Davies, P. 2005. Pea anther culture: callus initiation and production of haploid plants. In Proceedings of the Australian Branch of the IAPT\&B. Perth, Western Australia, 21-24th September, pp 180-186.

Touraev, A., Indrianto, A., Wratchko, I., Vicente, O., HeberleBors, E. 1996. Efficient microspore embryogenesis in wheat (Triticum aestivum L.) induced by starvation at high temperature. Sexual Plant Reproduction 9: 209-215. 